\title{
Comportamiento del aluminio desnudo y anodizado en atmósferas con grados de contaminación muy diferentes
}

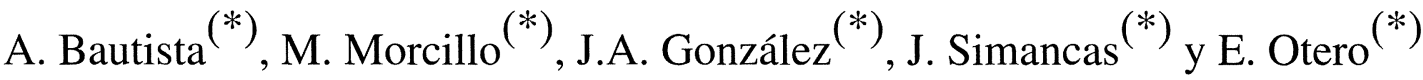

\begin{abstract}
Resumen En la presente investigación, comparando las respuestas de muestras de aluminio desnudas y anodizadas, expuestas a 12 atmósferas diferentes, se analiza la repercusión del anodizado y sellado del aluminio en el comportamiento frente a la corrosión atmosférica. Se demuestra que la anodización y sellado del aluminio elimina el riesgo de corrosión por picaduras que afecta a estos materiales en atmósferas marinas, sin que el espesor del recubrimiento resulte determinante, al menos, en el primer año de exposición.
\end{abstract}

Palabras clave: Aluminio desnudo. Anodización y sellado. Exposición atmosférica. Contaminación. Durabilidad.

\section{Behaviour of unprotected and anodized aluminium in atmospheres with very different polution degrees}

\begin{abstract}
In the present research, the responses of unprotected and anodized aluminium specimens are compared after they have been exposed to 12 different atmospheres. The significance of the anodizing and the sealing on the atmospheric corrosion behaviour is analysed. The results show that the anodizing and sealing of aluminium exclude the risk of pitting corrosion in marine atmospheres and that the oxide thickness is not determining, at least during the first year of exposure.
\end{abstract}

Keywords: Unprotected aluminium. Anodizing and sealing. Atmospheric exposure. Pollution. Durability.

\section{INTRODUCCIÓN}

De todos los metales muy electronegativos sólo al cromo y al aluminio les corresponde una relación de Pilling y Bedworth $>1$ (1). Su elevada afinidad con el oxígeno los capacita para desarrollar capas de óxido, ya a temperatura ambiente, a la que la favorable relación de Pilling y Bedworth proporciona excelentes características de adherencia, continuidad y resistencia a la corrosión.

Cuando se exponen al aire superficies recientes de aluminio, se forma, pues, una capa protectora de óxido extremadamente fina, en torno a 2,5 nm (2). Aunque no ha sido probado, se cree que esta capa de óxido existe en varios grados de hidratación $\left(\mathrm{Al}_{2} \mathrm{O}_{3} \times \mathrm{H}_{2} \mathrm{O}\right)$, dependiendo de las condiciones de

(*) Centro Nacional de Investigaciones Metalúrgicas, CENIM (CSIC), Avda. de Gregorio del Amo, 8. 28040-Madrid (España). exposición, especialmente la humedad relativa y la temperatura. En ambientes naturales, la reacción normal de corrosión implica la combinación con el agua para formar hidróxido de aluminio e hidrógeno (2):

$$
2 \mathrm{Al}+6 \mathrm{H}_{2} \mathrm{O} \longrightarrow 2 \mathrm{Al}(\mathrm{OH})_{3}+3 \mathrm{H}_{2}
$$

constituyendo, incluso sin el concurso de oxígeno libre, una película tan resistente a la corrosión que garantiza el comportamiento de las aleaciones de aluminio como materiales pasivos en los medios naturales: atmósfera, agua dulce, agua de mar y suelos.

Cuando la corrosión ocurre en presencia de ciertos contaminantes, es localizada -corrosión por picaduras, intergranular, corrosión bajo tensiones o exfoliación- siendo, con marcada diferencia, la corrosión por picaduras la más frecuente. En tales situaciones, la penetración máxima de las picaduras en función del tiempo se ajusta a expresiones de tipo semilogarítmico (3 y 4): 


$$
P_{\text {máx }}=a \log t+b
$$

donde $a$ es una constante, $b$, la penetración máxima de las picaduras durante el primer año y $t$, el tiempo de exposición en años (4). Tanto $a$ como $b$ dependen del tipo de aleación y la corrosividad de la atmósfera.

Contra el riesgo de corrosión localizada del aluminio y sus aleaciones se lucha esencialmente, por lo que a la exposición atmósférica se refiere, a través de la anodización de tales materiales, operación que multiplica el espesor de la película natural de óxido que se forma en la atmósfera por un factor próximo a $10^{4}(2)$, con la peculiaridad de desarrollar, en su parte más externa, una estructura porosa que potencia extraordinariamente sus aplicaciones decorativas, pero que exige una operación adicional de sellado para lograr la máxima resistencia a la corrosión, y garantizar la durabilidad en ambientes agresivos.

En la investigación presente se pretende esclarecer hasta qué punto la anodización y sellado representa una solución a los problemas de corrosión localizada de las aleaciones de aluminio en atmósferas marinas e industriales.

\section{METODOLOGÍA EXPERIMENTAL}

En las estaciones de ensayos naturales del proyecto iberoamericano PATINA (5), relacionadas en la tabla I, se expusieron probetas de aluminio de

TABLA I.- Características ambientales y corrosividad hacia el aluminio de las estaciones de ensayo de exposición atmosférica. Información disponible de estudios previos, excepto los datos de corrosión obtenidos en este estudio

TABLE I.- Behaviour of unprotected and anodized aluminium in atmospheres with very different pollution degrees

\begin{tabular}{|l|l|l|l|l|l|l|}
\hline Estaciones & $\begin{array}{l}\mathrm{T}^{\mathrm{a}} \\
\text { med. } \\
{ }^{\circ} \mathrm{C}\end{array}$ & $\begin{array}{l}\mathrm{HR} \\
\mathrm{med} \\
\%\end{array}$ & $\begin{array}{l}\mathrm{TDH} \\
\text { f. anual }\end{array}$ & $\begin{array}{l}\mathrm{SO}_{2} \\
\mathrm{mg} / \\
\mathrm{m}^{2} \mathrm{~d}\end{array}$ & $\begin{array}{l}\mathrm{Cl} \\
\mathrm{mg} / \\
\mathrm{m}^{2} \mathrm{~d}\end{array}$ & $\begin{array}{l}\mathrm{Corr} \\
\mathrm{g} / \mathrm{m}^{2} \mathrm{a}\end{array}$ \\
\hline Pardo & 15,0 & 55 & 0,366 & 6,4 & 3,9 & 0,13 \\
Esmeraldas & 26,8 & 78 & 0,710 & 16,5 & 2,7 & 0,38 \\
Panama & 26,9 & 71 & 0,572 & 21,7 & 9,8 & 0,36 \\
Lumiar & 16,1 & 78 & 0,135 & 22,6 & 26,7 & - \\
Tablazo & 27,7 & 77 & 0,504 & 6,0 & 53,7 & 0,65 \\
Lima & - & - & 0,660 & 14,0 & 54,9 & 5,2 \\
La Voz & - & - & 0,423 & 9,5 & 567,7 & 1,31 \\
Viriato & 24,8 & 79 & 0,474 & 42,4 & 919,3 & 2,42 \\
\hline
\end{tabular}

pureza comercial, 99,5\% Al. Otra serie de muestras se anodizó en solución de ácido sulfúrico del $18 \%$ en masa, a $20^{\circ} \mathrm{C}$ y $1,5 \mathrm{~A} / \mathrm{dm}^{2}$, durante distintos tiempos, de manera que resultaron capas de anodizado de 6,18 y $32 \mu \mathrm{m}$, cubriendo el margen de espesores usual en aplicaciones arquitéctónicas. Las probetas anodizadas se sellaron durante $60 \mathrm{~min}$ en agua destilada a ebullición, y se expusieron en las mismas estaciones de ensayo que las muestras de aluminio desnudo. En el curso de la investigación, se utilizaron técnicas gravimétricas, electroquímicas, los ensayos normalizados de control de calidad del sellado y las microscopías óptica y electrónica de barrido.

\section{RESULTADOS EXPERIMENTALES}

En la figuras 1 y 2 se reúnen las variaciones de masa experimentadas por las probetas de aluminio desnudo y anodizado, respectivamente, durante el

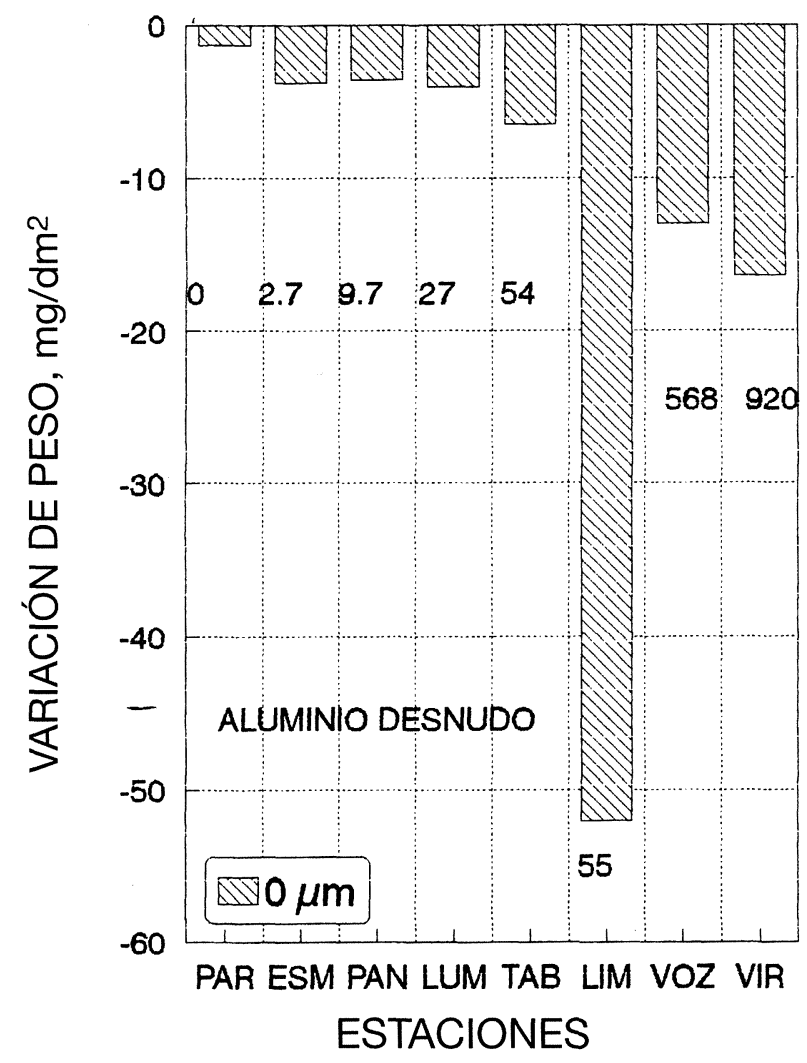

Fig. 1.- Variación de la masa de las probetas desnudas durante el primer año de exposición en las distintas estaciones de ensayo, situadas en la figura según el orden creciente de la contaminación en cloruros (Tabla I).

FIG. 1.- Mass change of bare aluminium after one year of exposure in different atmospheres. Test sites follow a crescent order of chloride deposition rate

(Table I). 


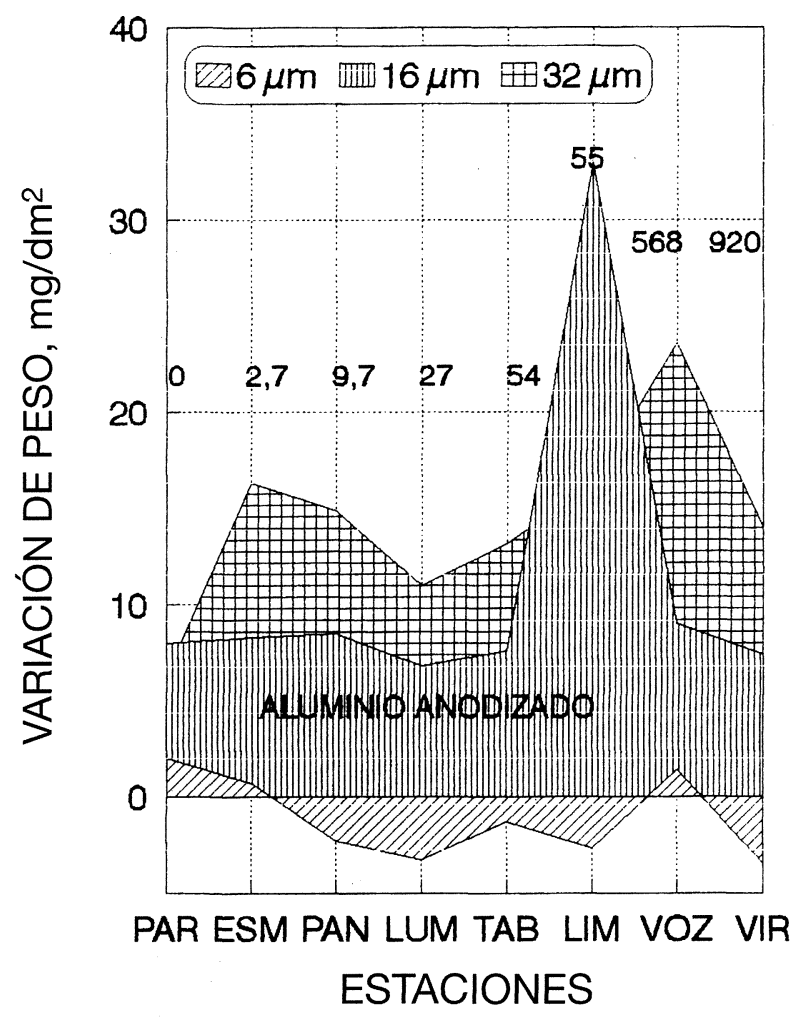

FIG. 2.- Variación de la masa de las probetas anodizadas durante el primer año de exposición en las distintas estaciones de ensayo. Se indica la contaminación por cloruros de cada estación en $\mathrm{mg} / \mathrm{m}^{2} \mathrm{~d}$.

FIG. 2.- Mass change of anodized aluminium after one year of exposure in different atmospheres. Test sites follow a crescent order of chloride deposition rate (Table I).

primer año de exposición en las distintas estaciones de ensayo. El aluminio desnudo sufre pérdidas de peso considerables en las estaciones de Lima, Viriato y La Voz, todavía significativas en la estación de Tablazo y muy pequeñas o despreciables en las restantes. Los anodizados de $6 \mu \mathrm{m}$ experimentan variaciones casi inapreciables de peso, de uno u otro signo, durante la exposición, mientras que aumentan por término medio unos $7 \mathrm{mg} / \mathrm{dm}^{2}$ los de $18 \mu \mathrm{m}$ y unos $15 \mathrm{mg} / \mathrm{dm}^{2}$ los de mayor espesor, de $32 \mu \mathrm{m}$, sin que el tipo de atmósfera tenga influencia apreciable el primer año de exposición.

En la figura 3, que muestra los diagramas de Bode correspondientes a anodizados de $32 \mu \mathrm{m}$ de espesor, antes de la exposición y después de 1 año en la atmósfera de Viriato, la de mayor contaminación en $\mathrm{Cl}^{-}$. Puede comprobarse que, con el tiempo, aumenta la impedancia en todo el campo de frecuencias, lo que equivale, en último término, a una mejora en la calidad de los anodizados (6).

A partir de diagramas de impedancia como el de la figura 3, pueden estimarse, según se esquematiza en ella, las características eléctricas de los recubri-

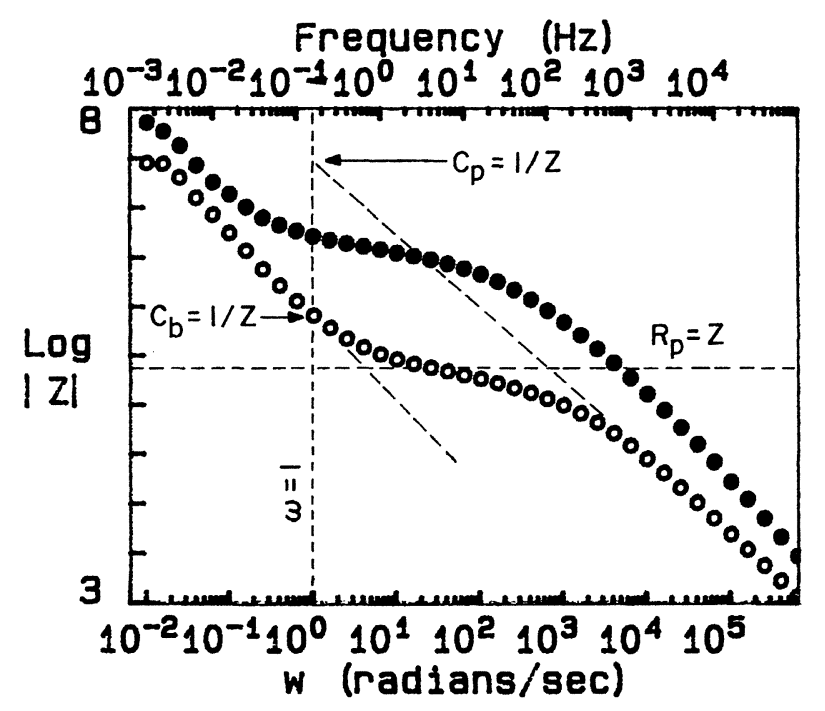

FIG. 3.- Diagramas de impedancia de las capas anódicas de $32 \mu \mathrm{m}$ antes (o) y después $(\bullet$ ) de un año de exposición en la estación de Viriato.

FIG. 3.- Impedance diagrams of $32 \mu \mathrm{m}$ anodized aluminium before $(o)$ and after $(\bullet)$ one year of exposure in Viriato test site.

mientos, que definen su integridad y calidad. En la tabla II se ofrecen tales parámetros, concretamente la resistencia y la capacidad de la capa porosa, $R_{\mathrm{p}}$ y $C_{\mathrm{p}}$, y la capacidad de la capa barrera, $C_{\mathrm{b}}$, para estaciones de contaminaciones muy dispares, junto a los resultados de los ensayos de la gota de colorante y la admitancia a $1 \mathrm{kHz}$, utilizados en la práctica industrial para medir rutinariamente la calidad de

TABLA II.- Ensayos normalizados y parámetros electroquímicos de las capas anódicas recién selladas y tras un período de exposición de 1 año en las distintas estaciones de ensayo

TABLE II.- Results of standard and electrochemical tests on anodic layers, both laboratory fresh postsealing and after one year of atmospheric exposure in different atmospheres

\begin{tabular}{|l|c|l|c|c|c|}
\hline Fichero & $\begin{array}{c}\mathrm{R}_{\mathrm{p}} \\
\mathrm{k} \Omega \mathrm{cm}^{2}\end{array}$ & $\begin{array}{c}\mathrm{C}_{\mathrm{b}} \\
\mu \mathrm{F}\end{array}$ & $\begin{array}{c}\mathrm{C}_{\mathrm{p}} \\
\mathrm{nF}\end{array}$ & G.C. & $\begin{array}{c}\text { Adm. } \\
\mu \mathrm{F}\end{array}$ \\
\hline LAB11D & 248 & 0,56 & 8,07 & 0,5 & 8 \\
LAB21D & 375 & 0,42 & 4,60 & 0,5 & 18 \\
LAB31D & 211 & 1,37 & 28,2 & 0,5 & 24 \\
\hline ESM11A & 1.820 & 2,5 & 7,16 & 0 & 2 \\
ESM21A & 2.490 & 0,47 & 1,14 & 0 & 4 \\
ESM31A & 5.410 & - & 0,93 & 0 & 6 \\
\hline VIR11A & 2.990 & - & 4,54 & 0,5 & 2 \\
VIR21A & 4.840 & - & 1,10 & 0,5 & 5 \\
VIR31A & 6.530 & 0,72 & 1,82 & 0,5 & 7 \\
\hline
\end{tabular}

LAB - laboratorio, ESM - Esmeraldas, VIR - Viriato

1,2 y $3=6,16$ y $32 \mu \mathrm{m}, 1 \mathrm{D}-1$ día, $1 \mathrm{~A}-1$ año 
las capas anódicas (7). Curiosamente, el efecto del grado de contaminación ambiental apenas incide en las respuestas de los anodizados.

Si que incide, en cambio, la contaminación en el comportamiento de las probetas de aluminio desnudo, cuyo aspecto parece impecable después de 1 año de exposición, una vez eliminados los depósitos de suciedad, siempre que la contaminación por $\mathrm{Cl}^{-}$no alcance $30 \mathrm{mg} / \mathrm{m}^{2} \mathrm{~d}$ (estación de Lumiar (Tabla I), de acuerdo con los resultados anteriores obtenidos en el proyecto iberoamericano MICAT (8). La situación parece comenzar a cambiar concretamente en la estación de Tablazo, con $54 \mathrm{mg} / \mathrm{m}^{2}$ $\mathrm{d}$ de $\mathrm{Cl}^{-}$. El ataque en forma de picaduras se acrecienta al pasar a las estaciones de La Voz y Viriato, conforme lo hace la contaminación, que pasa a 568 y $920 \mathrm{mg} / \mathrm{m}^{2} \mathrm{~d}$ de $\mathrm{Cl}^{-}$(Tabla I). En la estación de Lima, cuyo comportamiento constituye una excepción, debido a una contaminación elevadísima en partículas carbonosas, que constituyen pilas galvánicas con la superficie del aluminio, aparecen numerosas ampollas asociadas a las picaduras (Figs. 4 y 5).

\section{DISCUSIÓN}

\subsection{Comportamiento del aluminio sin anodizar}

La resultados de la figura 1 confirman la experiencia previa (2-4), en el sentido de demostrar la excelente resistencia a la corrosión del aluminio en los ambientes naturales no contaminados, así como su susceptibilidad a la corrosión localizada en presencia de iones despasivantes, como los cloruros de las atmósferas marinas.

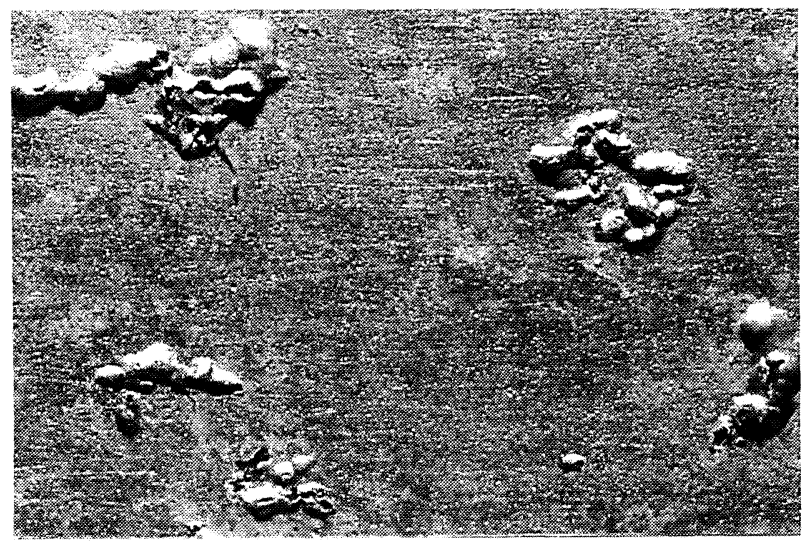

FIG. 4.- Micrografía, obtenida con el MEB, de la probeta de aluminio desnudo expuesta durante 1 año en Lima, mostrando corrosión localizada en forma de ampollas y picaduras.

FIG. 4.- SEM micrograph of bare aluminium after one year of exposure in Lima test site. Localized blisters and pits are shown.

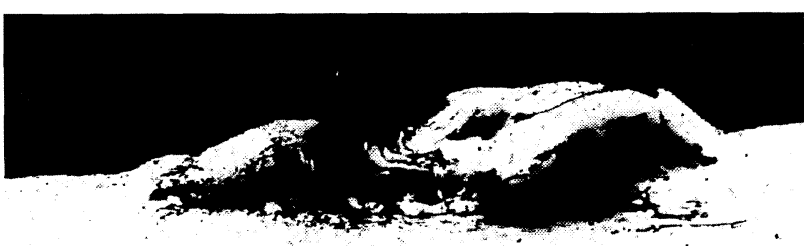

Fig. 5.- Corte transversal de una de las ampollas formadas en el $\mathrm{Al}$ sin anodizar en la estación de Lima.

\section{FIG. 5.- Cross section in a blister formed on bare aluminium in Lima test site.}

Concretamente, el aluminio desnudo se comporta como un material pasivo y no muestra signos de ataque localizado en ninguna de las estaciones atmosféricas con una contaminación en $\mathrm{Cl}^{-}<30$ $\mathrm{mg} / \mathrm{m}^{2} \mathrm{~d}$. La penetración de la corrosión es del orden de $0,1 \mu \mathrm{m} /$ año tan sólo (Fig. 1 y Tabla I). En cambio, en todas las estaciones con contaminación en $\mathrm{Cl}^{-}>50 \mathrm{mg} / \mathrm{m}^{2} \mathrm{~d}$, el aluminio sin anodizar sufre ataque localizado en forma de picaduras, tanto más intenso cuanto más elevado es el grado de contaminación (Fig. 1).

Aparte de la contaminación en $\mathrm{Cl}^{-}$y $\mathrm{SO}_{2}$, el depósito de partículas carbonosas tiene un efecto perjudicial muy acusado, como demuestra el comportamiento especial en la estación de Lima, a causa de la formación de pilas galvánicas muy activas entre un material muy electronegativo (aluminio) y otro muy electropositivo (carbono) (Figs. 4 y 5).

\subsection{Comportamiento del aluminio anodizado}

Según demuestran los resultados obtenidos, una anodización correcta parece representar una solución adecuada a los problemas de corrosión localizada de las aleaciones de aluminio en atmósferas muy contaminadas, pues no se ha detectado en ningun caso sintomatología visible de corrosión después de un año de exposición.

El proceso de envejecimiento supone una mejora de todos los índices de calidad de las capas anódicas (Tabla II), como se había comprobado previamente en la atmósfera de Madrid (5), sin que importe su espesor, dentro de los habituales en aplicaciones arquitectónicas, ni el grado de contaminación de las estaciones de ensayo (Figs. 2 y 3 y Tabla II). 


\section{Agradecimiento}

Los autores de este trabajo desean agradecer a $\mathrm{S}$. Flores (Perú), E. Almeida (Portugal), M. Sánchez (Panamá), J. Peña (Ecuador), M. Marrocos (Brasil), S. Rivero (Uruguay), O.T. de Rincón (Venezuela), F. Corvo (Cuba) y B. Rosales (Argentina) la exposición de los materiales en sus estaciones de ensayo.

\section{REFERENCIAS}

(1) Pilling, N.B. y Bedworth, R.E. J. Inst. Met., 29, 1923: 529.

(2) Goddard, H.P. Mater. Perf. 20 (7), 1981: 9.
(3) Feliu, S. y Morcillo, M. Corrosión y protección de los metales en la atmósfera. Ed. Bellaterra. Barcelona, 1982.

(4) Morcillo, M. y Feliu, S. Mapas de España de corrosividad atmosférica. Cap. 4. Eds. M. Morcillo y S. Feliu. Programa CYTED 1993. Proyecto MICAT.

(5) Morcillo, M. y col. $5^{\circ}$ Cong. Iberoam. Corr. Prot. Puerto de la Cruz, Tenerife, 22-27 oct., 1995.

(6) Lizarbe, R., Gonzalez, J.A., Otero, E. y Lopez, V. Aluminium, 69, 1993: 548.

(7) Normas UNE 38-16, 38-017 y 38-18.

(8) Morcillo, M. y Almeida, E. Corrosión atmosférica del aluminio, en Corrosión y protección de metales en las atmósferas de Iberoamérica, Eds. M. Morcillo, E. Almeida, B. Rosales, J. Urruchurtu y M. Marrocos, CYTED (Madrid), en prensa. 